\title{
Single port robotic assisted reconstructive urologic surgery-with the da Vinci SP surgical system
}

\author{
Mubashir Shabil Billah, Michael Stifelman, Ravi Munver, Johnson Tsui, Gregory Lovallo, \\ Mutahar Ahmed
}

Hackensack University Medical Center, Hackensack Meridian School of Medicine at Seton Hall University, NJ, USA

Contributions: (I) Conception and design: M Stifelman, MS Billah; (II) Administrative support: M Stifelman; (III) Provision of study materials or patients: M Stifelman, R Munver, G Lovallo, M Ahmed; (IV) Collection and assembly of data: MS Billah, J Tsui; (V) Data analysis and interpretation: MS Billah, M Stifelman; (VI) Manuscript writing: All authors; (VII) Final approval of manuscript: All authors.

Correspondence to: Mubashir Shabil Billah, MD; Michael Stifelman, MD. Hackensack University Medical Center, Hackensack Meridian School of Medicine at Seton Hall University, 30 Prospect Ave, Hackensack, NJ 07601, USA. Email: msb274@njms.rutgers.edu; michael.stifelman@hackensackmeridian.org.

Background: Single port (SP) robotic assisted laparoscopic surgery was approved by the FDA for urologic surgery and clinically available in 2018. This new robotic system enables a camera and 3 separate instruments, with fully wristed motions, to be placed through a single $25 \mathrm{~mm}$ port. This system was designed to perform complex surgery in narrow deep spaces making it very suitable for complex urinary tract reconstruction surgery. This paper will describe our early experience of using the SP system for several types of urinary reconstruction procedures and will present our lessons learned, surgical philosophy to using the SP and early data. As with all new technologies, there is an associated learning curve and nuances to be discovered and overcome.

Methods: The da Vinici SPTM surgical system was acquired and delivered to at our institution in January 2019. Five high volume robotic urologic surgeons at our institutions underwent certification with the da Vinci SP ${ }^{\mathrm{TM}}$ and have been adding this technology into their armamentarium. Almost all cases were recorded for quality improvement initiatives and evaluated with the goal of creating standard operating procedures in terms of access, steps of procedure and minimizing pit falls. Data from all patients undergoing SP urinary tract reconstruction that were entered into our prospective institutional database were reported.

Results: From 1/2019 to 8/2019 we have performed 71 urologic SP cases with the SP of which 18 were for urinary tract reconstructive procedures. These cases included 15 pyeloplasties, 1 buccal mucosa ureteroplasty, 1 ureteral implant and 1 repair of vesico-vaginal fistula. This paper outlines our standard operating procedures for table positioning, port placement, access and surgical steps for these complex SP cases. Our early data suggests that use of the SP system for urinary reconstruction is safe and reproducible.

Conclusions: The SP robotic surgical system has the potential to be used for nearly all robotic urologic reconstructive procedures. Advantages include a superior cosmetic result and ability to access all surgical quadrants without re-docking or repositioning. Limitations include no near infrared fluorescence imaging, smaller working space and slightly increased difficulty with retraction. We believe these obstacles will be overcome with time and experience. The da Vinci SP ${ }^{\mathrm{TM}}$ surgical system, in our initial experience, appears to be as safe and effective as its multiport counterpart for reconstructive surgeries.

Keywords: Reconstruction; pyeloplasty; ureteral reimplantation; ureteroplasty; vesicovaginal fistula

Submitted Sep 16, 2019. Accepted for publication Nov 11, 2019.

doi: $10.21037 /$ tau.2020.01.06

View this article at: http://dx.doi.org/10.21037/tau.2020.01.06 


\section{Introduction}

Laparoendoscopic single-site surgery (LESS) is a minimally invasive surgery technique developed in an effort to minimize port-related complications and reduce recovery time and postoperative pain while achieving improved cosmesis $(1,2)$. Due to the highly challenging nature of this technique even in expert hands, despite demonstrated feasibility in urologic surgery (3), the adoption of LESS has not been widespread due to its steep learning curve and the more recent adoptions of robotic assisted laparoscopy.

The da Vinci surgical system, which is the most commonly used robotic surgical system to date, first received FDA approval in 2000 and has since undergone several iterations as the technology continues to advance. Intuitive Surgical Inc. released the latest version of the DaVinci system in late 2018 , the da Vinci $\mathrm{SP}^{\circledR}$ surgical system.

The new system uses a single $25 \mathrm{~mm}$ trocar to introduce three, multi-jointed, wristed instruments and the first ever fully wristed 3D HD camera (4). The new system allows for excellent internal range of motion which offers the urologic community a more technically feasible approach to LESS. The single port (SP) system also enables dynamic external range of motion which allows the surgeon to change the target anatomy without having to redock the robot, remove instruments or change trocar position. The SP system uses the same surgeon console as the Da Vinci $\mathrm{X}$ and $\mathrm{Xi}$ systems that many urologists are already familiar with allowing surgeons to make a swift and easy transition to the latest robotics technology. With the SP surgical system, the issues of loss of triangulation and the constraints of laparoscopic straight arm surgery in LESS are addressed $(5,6)$.

The da Vinci SPTM platform is currently available at a limited number of institutions worldwide. However, feasibility has already been demonstrated in a variety of surgeries ranging from pyeloplasty to radical prostatectomy $(7,8)$. Herein, we describe our experience in using the da Vinci $\mathrm{SP}^{\mathrm{TM}}$ in a variety of reconstructive urologic procedures including pyeloplasty, ureteral reconstruction and buccal mucosa ureteroplasty, ureteral reimplantation, and vesicovaginal fistula repair. We offer our techniques, lessons learned, surgical philosophy and early data. Almost all cases were recorded for quality improvement initiatives and evaluated with the goal of creating standard operating procedures in terms of access, steps of procedure and minimizing pit falls.

\section{Methods}

\section{Access for SP surgery}

Access can be obtained via the umbilicus so that the SP cannula is positioned directly across from the target area (UPJ, upper and mid ureter) or caudad to the target area (reimplant, fistula). Occasionally we have used the lower quadrant along Pfannenstiel line with SP directed cephalad to target area (pediatric pyeloplasty). Selection of the initial incision needs to account for a minimum distance of 10 to $25 \mathrm{~cm}$ between the end of the cannula trocar and the target area. This allows for full deployment of the elbow and wristed joints of the robotic instruments and ability to operate with the surgical field minimizing collisions. For the majority of cases we used umbilical access except for the pediatric pyeloplasty patient and in select adults in which we have placed our incision along the Pfannenstiel crease.

We have adopted the use of the Mini GelPOINT ${ }^{\mathrm{Tm}}$ (Applied Medical, Rancho Santa Margarita, CA) (Figure 1). Use of this device provides the ability to "float" the trocar outside the body furthering the distance to the target area (Figure 1). This technique, of "floating the trocar" can make instrument exchanges challenging when the trocar is outside body and we suggest only performing instrument exchange with trocar intracorporeally. Instrument exchanges while floating the trocar is akin to instrument changes on the multiport without the use of a trocar to guide the instrument into the body. If the camera lens becomes foggy or bloody during the case, the camera needs to be removed and cleaned while the trocar is floating. When reinserting the camera, we recommend the assistant pulls up on the Alexis retractor or on the patient's fascia when reinserting the camera to create space for the camera to be reintroduced safely and cleanly.

The mini GelPOINT also allows the assistant to place their trocar through the device aside the robotic trocar. If utilizing this approach, we recommend that the trocars are placed through the GelSeal cap prior to attaching the cap to the Alexis retractor portion of the device. Our preference is to place two trocars through the GelSeal cap at opposite ends of the cap. We also avoid placing the SP cannula through the center of the GelSeal cap for added stability. Increased distance between the individual trocars allows for greater mobility and limits instruments clashing.

Some of our surgeons have not had success with the assistant working through the GelPoint or have preferred better assistant mobility and have moved to placing a $5 \mathrm{~mm}$ trocar in the lateral lower quadrant. When placing 

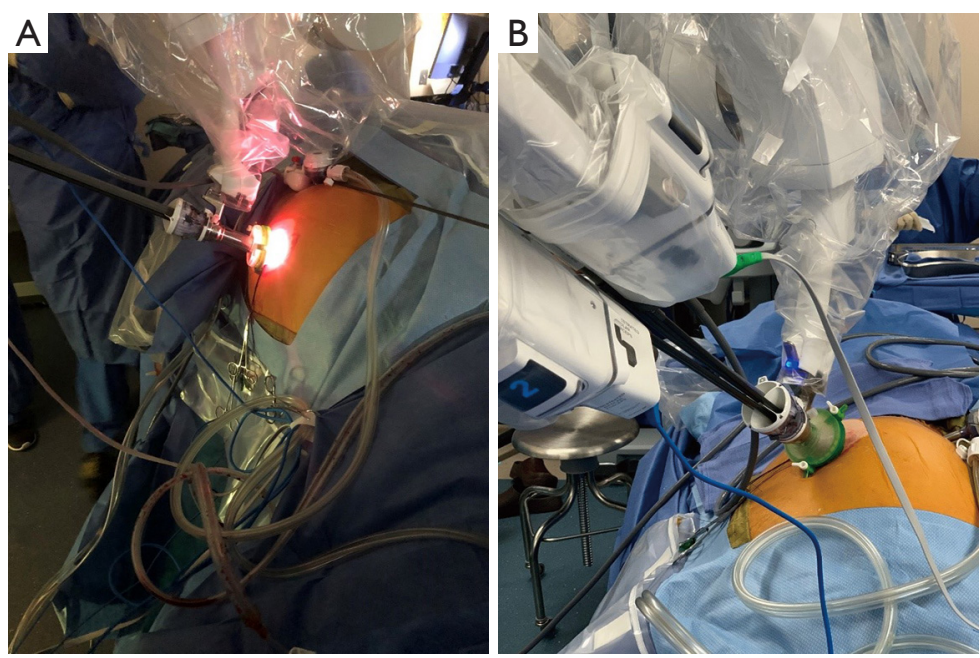

Figure 1 (A) Mini GelPOINT floated to increase distance and (B) trocar in GelPoint and not floated.
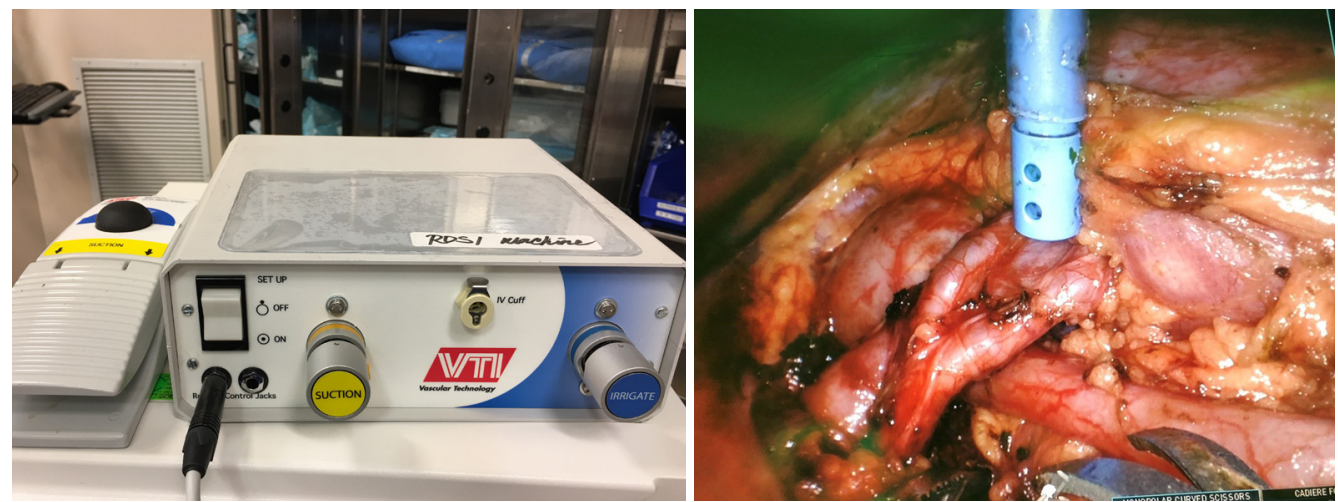

Figure 2 Remotely Operative Suction Irrigation System external set up and intraoperative use during pyeloplasty (Source: VTI Vascular Technology).

the assistant through the same incision as the robot, the assistant's mobility is restricted such that rigid suction, clip appliers and other instruments are of limited use. On a case by case basis and surgeon preference, a $5 \mathrm{~mm}$ trocar can be placed to improve mobility and utility of the assistant port.

Finally, we often use the miniGelPOINT to introduce a remote operated suction irrigation device (ROSI, VTI New Hampshire). This allows the surgeon to have a flexible suction/irrigation system in the operative field at all times, that can be grasped easily with a robotic instrument. We prefer to have the assistant control the suction and irrigation function through a foot pedal (Figure 2) (9).

For all cases we have used the $5 \mathrm{~mm}$ Airseal trocar placed either through the GelPOINT or as an assistant port as this provides stable pneumoperitoneum and allows us to run the pneumoperitoneum at 10-12 $\mathrm{mmHG}$, Decreasing the pneumoperitoneum may potentially further decrease postoperative complications (10).

\section{Pyeloplasty}

Pyeloplasty was one of the first cases we transitioned to the SP robot. Ureteropelvic junction (UPJ) obstruction often affects younger patients who seek improved cosmetic outcomes. Pyeloplasty also requires a limited operative field which suits the SP robotic system and our institution has a vast experience with robotic multiport pyeloplasty $(11,12)$. Similar to our initial reports of robotic assisted pyeloplasty for UPJ obstruction the approach to SP robotic assisted pyeloplasty continues to be via a transperitoneal approach. 
We position the patient in a lateral decubitus position. In males we prep the phallus into the field to allow bladder access via cystoscope to check stent position intra-operatively. In female patients, we either check the stent after the case is complete or place legs in a modified lithotomy position with torso in a modified flank allowing us access to the urethra and to check stent position intraoperatively.

One important observation is that when approaching the kidney and upper ureter in these 2 positions the table should be raised one to two feet higher than with a multiport approach. The base of the robot where the instruments attach nearly reaches the floor as the arms are pointed towards the target anatomy, particularly when mobilizing the upper pole. If the robot is too close to the floor, instrument exchange is unnecessarily challenging, and sterility can be compromised.

Once robot is docked, there are several noticeable differences between the SP and multiport system in terms of console surgeon recognition. There is a new robotic schematic, known as the Navigator, at the bottom of the screen allowing the surgeon to identify the position of each instrument in relation to each other and troubleshoot collisions. The perspective of the Navigator (above, side, front) can be adjusted to surgeon preference at the console. Incorporation of this into the surgical procedure is critical as is using the articulating camera. The camera is the first fully elbowed 3D HD camera that can be positioned to a "cobra" configuration which is when the camera wrist is elbow is up and the wrist articulated down into the center of the workspace. The camera icon turns green when in cobra position. This configuration mimics a 30 degree down position providing excellent visualization.

In addition, there are now 3 separate ways to control the camera. The traditional way is to use the camera clutch pedal to move the camera. An additional method is to activate the camera adjust feature which moves just the camera wrist. Camera adjust is activated by depressing the camera pedal and then twisting your wrist on one hand as though you are turning the key on a car. Finally, the entire robot with the camera can be moved in synchrony which is called relocating.

The camera can be placed in either the 12 or 6 o'clock position, however for pyeloplasty we exclusively introduce the camera at the 12 o'clock position. During relocating, minor adjustments to camera position can be performed from the console. Our instrument configuration is based on a right-handed surgeon. For dissection we use a monopolar scissor in the right hand, bipolar forceps and Cadiere forceps in the 6 or 9 o'clock position depending on traction required. Typically, we place the traction instrument in our 9 o'clock position and the bipolar instrument at 6 o'clock and assign both to the left hand. These are easily interchanged depending on traction required. We also use Weck clips to help further retract peritoneum or Gerota's fascia to improve exposure of the operative field. The Weck clips are applied to the lateral abdominal wall along with the tissue that needs to be retracted.

After reflecting the colon and exposing the renal pelvis, traction with the Cadiere forceps is through a 60 'clock configuration. The renal pelvis and proximal ureter are dissected from the peri-renal adipose tissue being careful to avoid stripping ureteral adventitia in order to preserve blood supply. If performing a redo pyeloplasty, ensure that a formal ureterolysis is performed and all fibrotic tissue removed from proximal ureter and pelvis. The single use scissors are excellent for these tasks.

Multiple techniques exist for performing the pyeloplasty portion of the procedure, both dismembered and nondismembered. We almost exclusively use the AndersonHynes dismembered pyeloplasty due to its versatility, ability to manage a large hydronephrotic renal pelvis and transpose ureter anterior to crossing vessel when necessary (Figure 3). We typically spatulate the ureter with scissors in a cephalad position and the pelvis with scissor in caudad position (Figure 3). The, single scissor use prevents need for a Potts scissor (Figure 3). We use all the same steps to complete anastomosis with two needle drivers, retraction in the most cephalad position and 5.0 Vicryl or Monocryl on an RB-1 needle. For those that choose, a non-dismembered pyeloplasty may be performed as well using similar surgical principles as above.

\section{Ureteroureterostomy/buccal mucosa ureteroplasty/ureteral reimplant}

Ureteral strictures are attributed to a variety of causes including, but not limited to infections, prior surgery, trauma, and malignancy (13). With the multiport technique location and port placement strategy was critical and based on location of stricture. The SP system has simplified our approach allowing us to exclusively place the SP trocar via the umbilicus and allow us to easily reach the upper to distal ureter and provide ability to locate omentum and create a flap. We advocate the use of the Mini GelPOINT ${ }^{\mathrm{TM}}$ for placement of the SP cannula as described above. If 

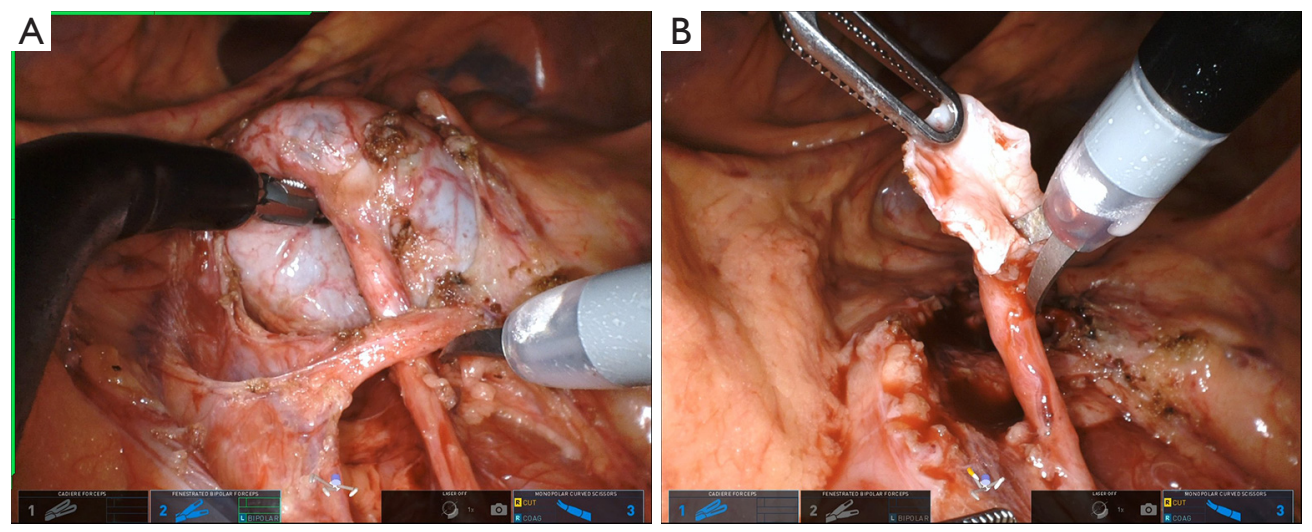

Figure 3 (A) Dissection of crossing vessel causing UPJ obstruction and (B) ureteral spatulation.

one chooses to use an assistant port, other than placed through gelpoint, we recommend placing the trocar within the contralateral lower quadrant for distal strictures and ipsilateral lower quadrant for proximal strictures. This placement of the assistant trocar in the lower quadrant may enable more assistant flexibility and provide cosmesis as the incision is hidden within the pant line of most individuals. It can also be used as a drain location. Patient positioning for upper and mid ureteral strictures is similar to pyeloplasty as described above. For distal ureteral reimplant and boari flap, patient is placed supine with access to the phallus in men and lithotomy in women for access to the urethra and bladder.

Instrument choice and configuration is similar to our pyeloplasty configuration. The Cadiere forceps provide a balance of grip strength while minimizing tissue damage and used primarily for traction purposes. The Cadiere may be assigned to surgeon's right hand or left hand and placed anywhere within the trocar based on surgeon's requirements. The fenestrated bipolar forcep is controlled by the surgeon's left hand for manipulation, traction, dissection and hemostasis. The monopolar curved scissor is controlled by the surgeon's right hand similar to a pyeloplasty and a multiport approach. Camera remains at the 12 o'clock position. This provides a similar feel and muscle memory to a multiport approach.

Different than a multiport, we find ourselves changing and considering optimum instrument position in relation to traction and mobility during SP ureteral reconstruction cases and relying on the Navigator to maximize our mobility and access to the operative field. Changing instrument positions during different portions of a case can greatly assist progress. For example, when mobilizing the colon, we prefer the Caidere in the 6 o'clock position for traction. While dissecting the ureter, we place the Cadiere in either the 3 or 9 o'clock position.

Another difference is that when performing complex ureteral reconstructive procedures with the multiport we rely heavily on ICG and near infrared imaging to confirm perfusion to the ureteral anastomosis and when required the omental flap. The SP lacks the Firefly fluorescence system which we consider a limitation that we believe will be overcome shortly with improved SP camera technology.

We have not made any significant changes to the steps of uretero-urterostomy, buccal mucosa ureteroplasty, ureteral reimplant, boari flap or technique for omental wrap (Figure 4) and all have been described previously (14-20). As highlighted one must be vigilant of instrument configuration and placement, camera angle, schematic at bottom of screen, and new relocation pedal. Adjunct technology including ROSI, weck clips for traction, Magnetic retractors can all help to facilitate the exposure and surgery.

\section{Vesicovaginal fistula repair}

Vesicovaginal fistulas occur as the result of pelvic radiation, infection, malignancy, obstructed labor, or iatrogenic from prior surgery $(21,22)$. The primary goal of management in vesicovaginal fistulas is achieving healthy mucosa to mucosa closure with possible interposition of peritoneum or a graft (23). Our experience with multiport robotic transperitoneal vaginal fistula repair began in 2007 and have recently transitioned to SP cases starting 2019. For this utilization, where the target area is deep in the pelvis, working 

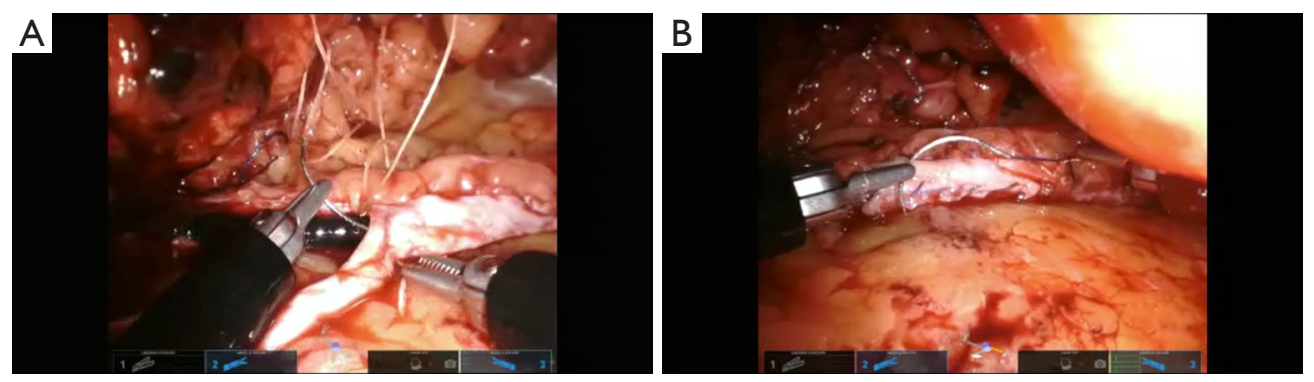

Figure 4 (A) Suturing buccal mucosa to ureter and (B) tying final knot after placement of buccal mucosa on ureter. Ureteroscope can be noted in the lumen of the ureter.

space is narrow and the operative field is small, the SP has proven to have all the correct characteristics and functionality.

Like most of our reconstructive procedures the SP is introduced through a periumbilical incision using a minigelPOINT as described previously. We always place the patient in dorsal lithotomy position for exposure to the vagina and urethra. The assistant trocar may be placed through the miniGelpoint aside the SP trocar which, in our early experience, is inconsistent and difficult for rigid suction mobility. Secondary to this difficulty we now use the ROSI (Figure 2) suction which allows for infinite angles and mobility for suctioning purposes as described previously. Another option is place an assistant trocar inferolateral to the periumbilical incision on the left or ride side based on surgeon preference.

The scope is placed at the 12 o'clock position the bipolar forceps is placed at the 9 o'clock position, the Cadiere forceps at the 3 o'clock position, and the monopolar curved scissors at the 6 o'clock position. With this setup, we have found that instruments do not need to be exchanged throughout the procedure which reduces operative time. When suturing with the SP system we utilize the needle drivers at the 6 and 9 o'clock position. On the multiport system, when tying knots, the wrist can be held at any angle and the instruments pulled laterally. One does not need to consider wrist angle. With the SP system, lateral movements are limited. To increase lateral distance that the suture can be pulled, we evert the tips of the needle driver and pull as far lateral as the SP system enables. This allows knots to be tied securely and especially important when closing the vaginal wall.

The major steps of the procedure do not differ from the multiport approach (24-26). Key elements begin with finding the posterior bladder and vaginal cuff. The plane between the vaginal cuff and posterior bladder is mobilized until the fistula tract is identified. Near the fistula tract, a cystotomy is performed and the fistula tract can easily be seen from within the bladder. The fistula is then excised and healthy tissue edges from the bladder mucosa and muscle and healthy vaginal tissue are brought together. Then mucosa to mucosa closure of the respective organs is performed in a tension free and watertight fashion. Interposition can be performed with omentum or peritoneum. A surgical drain can be brought out through the initial SP incision.

Some tips include when visualization of bladder closure is challenging, we have rotated the robotic arm 180 degrees and placed camera at the 60 'clock position allowing a better view and easier closure of the posterior bladder wall. For harvesting omentum the entire system can be relocated without changing patient position or redocking the robot and then used to bring omental flap into position. Early on we like having an extra assistant in the lateral lower quadrant to assist with suction, irrigation, traction and efficient passage of sutures.

\section{Results}

We have performed 18 robotic urinary reconstructive procedure of which 6 had complete information and IRB Consent (Tables 1,2).

\section{Conclusions}

As the boundaries of minimally invasive surgery are expanded, the introduction of the da Vinci SP ${ }^{\mathrm{TM}}$ robotic surgical system provides the most dramatic advancement in performing LESS (Figure 5). By providing a platform that operates similarly to the more widely adopted da Vinci ${ }^{\mathrm{TM}}$ multiport platform, the learning curve is drastically reduced. 

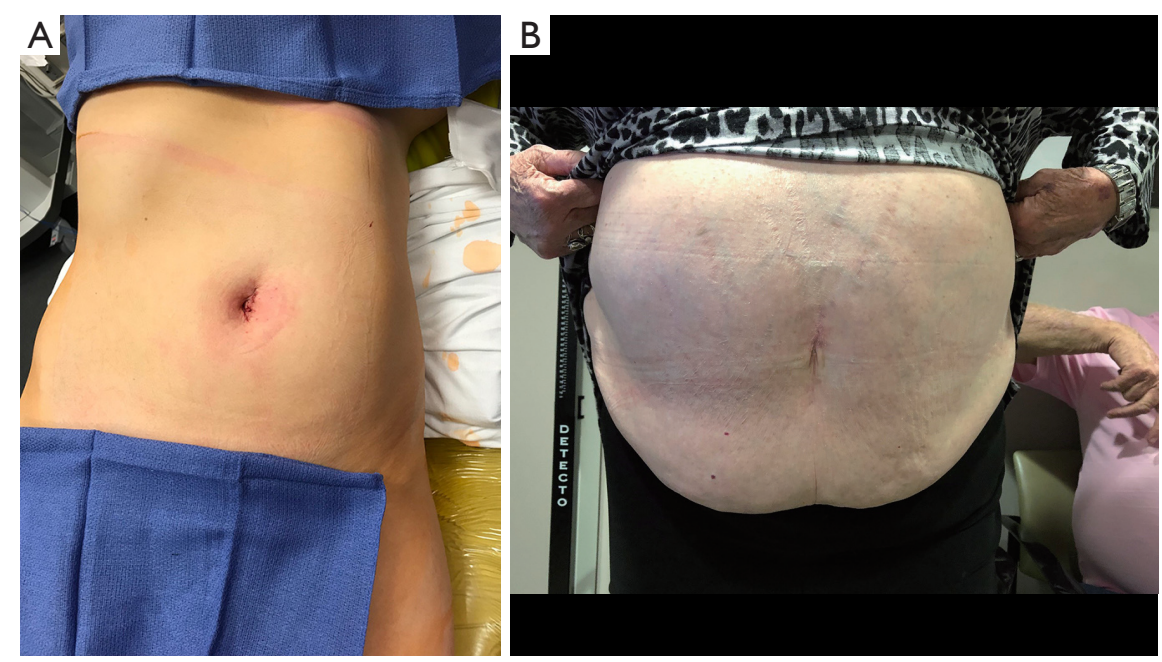

Figure 5 (A) As can be seen in the immediate postoperative period, the SP system enables smaller incisions and superior cosmesis, (B) excellent healing and cosmesis noted at follow-up visit.

Table 1 Single port reconstruction patient characteristics

\begin{tabular}{lccccccc}
\hline Pt & Sex & Height $(\mathrm{cm})$ & Weight $(\mathrm{kg})$ & BMI & Procedure & Etiology & Stricture length \\
\hline 1 & F & 170.2 & 101.6 & 35.1 & Ureteroplasty with buccal graft & Impacted Stone \\
2 & M & 185.4 & 74.8 & 21.8 & Pyeloplasty & Congenital & N/A \\
3 & F & 165.1 & 59 & 21.6 & Pyeloplasty & Congenital & Malignancy \\
4 & M & 177.8 & 78 & 24.7 & Distal ureterectomy & Congenital & N/A \\
5 & F & 165.1 & 80.7 & 29.6 & Pyeloplasty & Crossing Vessels \\
6 & F & 170.2 & 64.9 & 22.4 & Pyeloplasty and stone & 1.5 \\
\hline
\end{tabular}

Table 2 Single port reconstruction operating room data

\begin{tabular}{lcccccc}
\hline Pt & OR Time & EBL $(\mathrm{mL})$ & ASA score & Intra-Op Complications & Complications (Clavien 1-5) & Need to re-operate? \\
\hline 1 & 285 & 20 & 2 & None & None & None \\
2 & 157 & 50 & 2 & None & None & No \\
3 & 138 & 70 & 2 & None & None & No \\
4 & 128 & 10 & 2 & None & None & None \\
5 & 136 & 15 & 2 & None & No \\
\hline
\end{tabular}

EBL, estimated blood loss.

Herein, we present our initial experience with the da Vinci $\mathrm{SP}^{\mathrm{TM}}$ in urologic reconstructive surgery. Further studies in outcomes for procedures performed with the da Vinci SPтм may aid in more widespread adoption.

\section{Acknowledgments}

Study data were collected and managed using REDCap electronic data capture tool.

Funding: None. 


\section{Footnote}

Provenance and Peer Review: This article was commissioned by the Guest Editor (Ashok K. Hemal) for the series "Robotic-assisted Urologic Surgery" published in Translational Andrology and Urology. The article was sent for external peer review organized by the Guest Editor and the editorial office.

Conflicts of Interest: The series "Robotic-assisted Urologic Surgery" was commissioned by the editorial office without any funding or sponsorship. RM: Boston Scientific, Amniox Medical; MS: Conmed, Ethicon, Intuitive Surgical, Vascular Technology Incorporated. The other authors have no other conflicts of interest to declare.

Ethical Statement: The authors are accountable for all aspects of the work in ensuring that questions related to the accuracy or integrity of any part of the work are appropriately investigated and resolved. The study was approved by the Ethics Committee of Hackensack University Medical Center/Institutional Review Board (IRB number: Pro 2017-0476) and written informed consent was obtained from all patients.

Open Access Statement: This is an Open Access article distributed in accordance with the Creative Commons Attribution-NonCommercial-NoDerivs 4.0 International License (CC BY-NC-ND 4.0), which permits the noncommercial replication and distribution of the article with the strict proviso that no changes or edits are made and the original work is properly cited (including links to both the formal publication through the relevant DOI and the license). See: https://creativecommons.org/licenses/by-nc-nd/4.0/.

\section{References}

1. Box G, Averch T, Cadeddu J, et al. Nomenclature of natural orifice translumenal endoscopic surgery (NOTES) and laparoendoscopic single-site surgery (LESS) procedures in urology. J Endourol 2008;22:2575-81.

2. Gettman MT, Box G, Averch T, et al. Consensus statement on natural orifice transluminal endoscopic surgery and single-incision laparoscopic surgery: heralding a new era in urology? Eur Urol 2008;53:1117-20.

3. Autorino R, Cadeddu JA, Desai MM, et al. Laparoendoscopic single-site and natural orifice transluminal endoscopic surgery in urology: a critical analysis of the literature. Eur Urol 2011;59:26-45.

4. Intuitive Surgical I. Intuitive Surgical Announces Innovative Single Port Platform — the da Vinci SP® Surgical System. Intuitive Surgical [Internet] 2018 [cited 2019 Sep 1]. Available online: http://investor. intuitivesurgical.com/news-releases/news-release-details/ intuitive-surgical-announces-innovative-single-portplatform-da

5. Best SL, Donnally C, Mir SA, et al. Complications during the initial experience with laparoendoscopic single- site pyeloplasty. BJU Int 2011;108:1326-9.

6. Autorino R, Kaouk JH, Stolzenburg JU, et al. Current status and future directions of robotic single-site surgery: a systematic review. Eur Urol 2013;63:266-80.

7. Chang Y, Lu X, Zhu Q, et al. Single-port transperitoneal robotic-assisted laparoscopic radical prostatectomy (spRALP): Initial experience. Asian J Urol 2019;6:294-7.

8. Heo JE, Kang SK, Koh DH, et al. Pure single-site robotassisted pyeloplasty with the da Vinci SP surgical system: Initial experience. Investig Clin Urol 2019;60:326-30.

9. Stifelman MD, Mass A. \#WCE2014 - The use of a robotic remotely operated suction/irrigation (ROSI) system may lead to less post-operative blood loss after robotic pyeloplasty - Interview [Internet]. World Congress of Endourology 2014 [cited 2019 Sep 3]. Available online: https://www.urotoday.com/conference-highlights/globalcongress-on-bladder-cancer-2018/1622-conferences/ wce-2014/wce-2014-trauma-reconstruction/75097wce2014-the-use-of-a-robotic-remotely-operated-suctionirrigation-rosi-system-may-lead-to-less-post-operat

10. Rohloff M, Cicic A, Christensen C, et al. Reduction in postoperative ileus rates utilizing lower pressure pneumoperitoneum in robotic-assisted radical prostatectomy. J Robot Surg 2019;13:671-4.

11. Palese MA, Munver R, Phillips CK, et al. Robot-assisted laparoscopic dismembered pyeloplasty. JSLS 2005;9:252-7.

12. Niver BE, Agalliu I, Bareket R, et al. Analysis of roboticassisted laparoscopic pyleloplasty for primary versus secondary repair in 119 consecutive cases. Urology 2012;79:689-94.

13. Marien T, Bjurlin MA, Wynia B, et al. Outcomes of robotic-assisted laparoscopic upper urinary tract reconstruction: 250 consecutive patients. BJU Int 2015;116:604-11.

14. Stifelman MD. V1820: Robotic Pyeloplasty for the Treatment of Secondary UPJ Obstruction: Lessons Learned. J Urol 2007;177:605.

15. Stifelman MD, Hyams ES. V1056: Laparoscopic Doppler 
Technology: Applications in Laparoscopic Pyeloplasty, Radical and Partial Nephrectomy. J Urol 2007;177:349.

16. Stifelman M, Feliciano J. V860 robotic transmesenteric pyeloplasty of a pelvic kidney. J Urol 2011;185:e345.

17. Lipkin ME, Shah OD, Stifelman MD. Robotic-assisted ureteral re-implant and psoas hitch in the treatment of distal ureteral stricture. J Urol 2008;179:543.

18. Berger AD, Shah OD, Stifelman MD. V488: The Use of Rotobics in the Management of Mid Ureteral Obstruction. J Urol 2007;177:163.

19. Korets R, Hyams ES, Shah OD, et al. V485: RobotAssisted Laparoscopic Ureterocalicostomy. J Urol 2007;177:162.

20. Fenig DM, Berger AD, Kau E, et al. V752: RoboticAssisted Laparoscopic Ureteral Reimplantation. J Urol 2005;173:204.

21. Moses RA, Ann Gormley E. State of the Art for Treatment of Vesicovaginal Fistula. Curr Urol Rep 2017;18:60.

Cite this article as: Billah MS, Stifelman M, Munver R, Tsui J, Lovallo G, Ahmed M. Single port robotic assisted reconstructive urologic surgery-with the da Vinci SP surgical system. Transl Androl Urol 2020;9(2):870-878. doi: 10.21037/ tau.2020.01.06
22. Bodner-Adler B, Hanzal E, Pablik et al. Management of vesicovaginal fistulas (VVFs) in women following benign gynaecologic surgery: A systematic review and metaanalysis. PLoS One 2017;12:e0171554.

23. McKay E, Watts K, Abraham N. Abdominal Approach to Vesicovaginal Fistula. Urol Clin North Am 2019;46:135-46.

24. Agrawal V, Kucherov V, Bendana, et al. Robot-assisted Laparoscopic Repair of Vesicovaginal Fistula: A Singlecenter Experience. Urology 2015;86:276-81.

25. Martini A, Dattolo E, Frizzi J, et al. Robotic vesico-vaginal fistula repair with no omental flap interposition. Int Urogynecol J 2016;27:1277-8.

26. Kelly E, Wu MY, MacMillan JB. Robotic-assisted vesicovaginal fistula repair using an extravesical approach without interposition grafting. J Robot Surg 2018;12:173-6. 